\title{
Fatores comportamentais associados ao sobrepeso e à obesidade em escolares do Estado de Santa Catarina
}

\author{
Arlete Catarina Tittoni Corso* \\ Gilberto Veras Caldeira** \\ Giovanna Medeiros Rataichesck Fiates ${ }^{\star * \star}$ \\ Bethsáida de Abreu Soares Schmitz ${ }^{* * *}$ \\ Gabriela Dalsasso Ricardo***** \\ Francisco de Assis Guedes de Vasconcelos ${ }^{\star \star * * * *}$
}

\begin{abstract}
Foi realizado estudo transversal com 4.964 escolares do ensino fundamental do Estado de Santa Catarina para verificar associação entre as variáveis socioeconômicas e de atividade física com o sobrepeso e a obesidade, estes últimos diagnosticados a partir do Índice de Massa Corporal (IMC). Foram estimadas, por ponto e por intervalo com 95\% de confiança, as prevalências de sobrepeso e obesidade, variáveis de desfecho. As razões de prevalência foram obtidas pelo modelo de Poisson e ajustadas à renda familiar per capita, escolaridade dos pais, sexo e idade da criança. As prevalências de sobrepeso e obesidade foram $15,4 \%$ e $6,1 \%$, respectivamente, semelhantes entre sexo e categorias de idade. Após ajuste, as variáveis associadas com sobrepeso e obesidade foram: frequência semanal de esportes; número de horas sono/noite; e horas/dia no computador (fim de semana). Reduzida prática de esportes, menor duração de sono/noite e mais tempo no computador associam-se com sobrepeso e obesidade nestes escolares.
\end{abstract}

Palavras-chave: Criança. Estado nutricional. Escola. Excesso de peso.

\section{Introdução}

A elevada prevalência de sobrepeso e obesidade é um problema de saúde pública em escala mundial, que deixou de ser exclusivo dos países desenvolvidos, expondo as populações adulta e infantil ao desenvolvimento de morbidades como diabete mellitus, hipertensão, hiperlipidemia e doenças cardiovasculares (WHO,1998).
A transição nutricional verificada em diversas partes do mundo, caracterizada pela redução na prevalência dos déficits nutricionais e ocorrência mais expressiva de sobrepeso e obesidade não somente na população adulta, mas também em crianças e adolescentes, vem repercutindo sobre a situação de saúde e qualidade de vida destes grupos etários (BATISTA FILHO et al., 2008). Por isso, o diagnóstico precoce

\footnotetext{
* Programa de pós-graduação em Nutrição da Universidade Federal de Santa Catarina - UFSC.

** Graduação em Nutrição da Universidade Federal de Santa Catarina - UFSC.

*** Programa de pós-graduação em Nutrição da Universidade Federal de Santa Catarina - UFSC

**** Programa de Pós-Graduação em Nutrição da Universidade de Brasília - UnB.

***** Programa de pós-graduação em Nutrição da Universidade Federal de Santa Catarina - UFSC.

****** Programa de pós-graduação em Nutrição da Universidade Federal de Santa Catarina - UFSC.
} 
e as intervenções no período da infância e da adolescência vêm sendo recomendados para evitar o desenvolvimento de doenças crônicas não transmissíveis na idade adulta (WHO, 2004).

A obesidade é uma doença crônica caracterizada pelo acúmulo excessivo de energia, sob a forma de triglicérides, no tecido adiposo distribuído pelo corpo, enquanto o sobrepeso corresponde ao excesso de peso previsto para o sexo, altura e idade, de acordo com os padrões populacionais de crescimento, podendo representar ou não excesso de gordura corporal (LIMA et al., 2004).

A obesidade é resultante do desequilíbrio crônico entre a energia ingerida e a utilizada, de origem multifatorial, incluindo fatores socioeconômicos, biológicos, psicológicos e ambientais (OLIVEIRA et al., 2003). A transição nutricional caracterizada por um crescimento no consumo de alimentos ricos em açúcares e gorduras e pobres em fibras, associada ao sedentarismo decorrente da redução de atividades físicas e aumento do tempo gasto com TV e aparelhos eletrônicos entre outros, está impondo importantes mudanças no estilo de vida e, consequentemente, aumento na prevalência de sobrepeso e obesidade (OLIVEIRA et al., 2003).

Diversos pesquisadores têm procurado investigar variáveis socioeconômicas, ambientais e biológicas como fatores associados à ocorrência de sobrepeso e obesidade (WHO, 2004; GUMARÃES et al., 2006; BATISTA FILHO et al., 2008). A genética desempenha papel importante na determinação da massa corporal aumentada, porém, a prevalência de sobrepeso e obesidade nas últimas décadas vem sendo relacionada com as mudanças no ambiente onde vive a população (GUIMARÃES et al., 2006). Embora a prevalência de sobrepeso e obesidade em indivíduos de ambos os sexos, em diferentes faixas etárias e em diversos países do mundo possa ser considerada um fenômeno mundial e de natureza multifatorial, a redução nos níveis de atividade física usual e os hábitos alimentares inadequados, de forma isolada ou combinada, parecem ser os principais determinantes destes eventos (GUIMARÃES, et al., 2006).

Outras variáveis ambientais, relacionadas ao estilo de vida, também podem influenciar a prevalência de sobrepeso e obesidade, como o sedentarismos e o número de horas despendidas com o sono, ou diante da televisão ou do computador. A atividade física desempenha papel importante na prevenção das enfermidades crônicas não transmissíveis e diminui o risco de desenvolvimento de sobrepeso e de obesidade, atuando na regulação do balanço energético e preservando ou mantendo a massa magra em detrimento da massa gorda (DANADIAN et al., 2000).

Os avanços tecnológicos induziram as crianças a se tornarem menos ativas nas últimas décadas. Nos anos 1990, as pesquisas já relatavam relação positiva entre a inatividade, como o tempo gasto assistindo televisão, e o aumento da adiposidade em escolares (BAUGHCUM et al., 2000). O tempo gasto pela criança na frente de aparelhos eletrônicos, como televisão, computador e video game, resulta no sedentarismo, contribuindo para o aumento da prevalência de sobrepeso e de obesidade infantil. Por outro lado, as brincadeiras realizadas em casa e as atividades físicas na escola atuam na prevenção de sobrepeso e de obesidade (BAUGHCUM et al., 2000).

O número de horas de sono é uma variável importante, associada ao descontrole de ingestão alimentar e à obesidade (GUIMARÃES et al., 2006). A redução do número de horas de sono tornou-se um hábito comum na atualidade, determinada pelo modo de vida na sociedade moderna, sendo que a duração autorreportada de sono diminuiu de 1,5 a 2 horas nos Estados Unidos (CRISPIM et al., 2007). Estudos epidemiológicos correlacionam o tempo dedicado ao sono com a obesidade em diferentes populações, identificando o número de horas de sono como um fator predisponente para o aparecimento da obesidade (GRUPTA et al., 2002; SEKINE et al., 2002; CRISPIM et al., 2007).

Este artigo tem como finalidade analisar a associação entre sobrepeso e obesidade e as variáveis horas gastas com 
TV/computador, horas de sono e atividade física, em uma amostra de escolares de 6 a 10 anos matriculados em escolas de ensino fundamental do Estado de Santa Catarina.

\section{Método}

Os dados analisados no presente artigo são parte de um estudo transversal de base populacional, realizado entre junho de 2007 e maio de 2008, com escolares matriculados em escolas públicas e particulares de ensino fundamental, localizadas em oito municípios do Estado de Santa Catarina. Localizado na Região Sul do Brasil, o Estado possui, segundo dados do IBGE (2007), uma população de 5.866 .487 habitantes e um território de 95,4 mil km².

De acordo com dados do Censo Escolar do Ministério da Educação, em 2006 havia 4.007 escolas de ensino fundamental em Santa Catarina, sendo uma federal, 3.661 públicas (municipais e estaduais) e 345 particulares. ${ }^{1}$ Para o cálculo da amostra, foram excluídas a escola federal e aquelas que declararam não possuir escolares nas séries iniciais do ensino fundamental. Para a definição do número de escolas a serem investigadas, consideraram-se a dependência administrativa (pública e particular) e o número de escolares matriculados.

Para definição da amostra foi considerado o universo composto por 140.878 escolares de 569 escolas públicas e particulares de oito municípios-polo que possuíam o maior número de escolares matriculados nas séries iniciais do ensino fundamental: Chapecó e Joaçaba, no oeste do Estado; Blumenau, Jaraguá do Sul e Lages, no centro; Criciúma, Florianópolis e Joinville, no litoral.

O número de escolas a ser investigado foi calculado de modo a garantir um erro amostral de no máximo 6 pontos percentuais, para mais ou para menos, para cada um dos estratos de interesse. Assim, a amostra final foi composta por 347 escolas situadas em áreas urbanas e rurais, sendo 266 públicas e 81 particulares.
A pesquisa foi planejada para obtenção de dados de todos os escolares matriculados entre a $1 \underline{\text { a }}$ e a $4 \underline{\text { a }}$ série de todas as escolas selecionadas, sendo que a amostra foi aleatória com partilha proporcional à série. A amostra final ficou na dependência do número de escolares matriculados na série sorteada em cada escola e também do retorno do Termo de Consentimento Livre e Esclarecido por parte dos pais ou responsáveis, totalizando 5.686 escolares autorizados a participarem da pesquisa.

A equipe responsável pela coleta dos dados, composta por oito nutricionistas, foi treinada com base em um protocolo de padronização dos procedimentos de coleta de dados, previamente estabelecido no sentido de minimizar os possíveis erros intra e interavaliadores. Não foi determinado o erro técnico de medição entre a equipe de coleta, mas $10 \%$ da amostra dos escolares foi medida em duplicata para o controle da qualidade das medidas antropométricas.

Dados sobre região, dependência administrativa da escola, idade e sexo foram obtidos na secretaria das escolas e repassados para uma ficha de identificação do escolar. Já as informações sobre meio de locomoção até a escola, prática de esportes, horas/dia de televisão/computador, horas de sono/noite, renda familiar per capita e escolaridade dos pais foram coletados por meio de questionário enviado aos pais ou responsáveis pela criança.

Os dados antropométricos de peso e estatura foram obtidos de acordo com procedimentos descritos no Anthropometric Standardization Reference Manual (LOHMAN et al., 1991). As medidas de peso e estatura foram utilizadas para o cálculo do índice de Massa Corporal - IMC. As variáveis de desfecho são o sobrepeso e a obesidade, classificados de acordo com IMC por sexo e idade, segundo os pontos de corte propostos por Cole e colaboradores (2000), recomendados pela International Obesity Taskforce - IOTF, com base nos valores de desfecho de sobrepeso (valores

\footnotetext{
${ }^{1}$ Disponível em: <http://www.inep.gov.br/censo/basica/dataescolabrasil/>
} 
equivalentes ao IMC maior que $25 \mathrm{~kg} / \mathrm{m}^{2}$ e menor que $30 \mathrm{~kg} / \mathrm{m}^{2}$ de adultos) e obesidade (valores equivalentes ao IMC igual ou maior que $30 \mathrm{~kg} / \mathrm{m}^{2}$ de adultos).

As variáveis independentes relativas à atividade física são: meio de locomoção ativo para a escola; prática de esportes extraescolar; frequência semanal de esportes; horas por dia de televisão durante a semana e no final de semana; horas por dia no computador durante a semana e no final de semana; horas de sono por noite; sexo e idade da criança. As variáveis de controle foram sexo e idade da criança, renda mensal per capita e escolaridade dos pais.

As estatísticas foram estimadas por ponto e por intervalo com $95 \%$ de confiança (IC 95\%). Nas análises descritivas, as variáveis categóricas foram representadas por percentagens, enquanto as quantitativas foram expressadas por valores médios e dos desvios padrão (dp) e as qualitativas, por frequências absolutas e relativas, segundo o estado nutricional das crianças. Para variáveis quantitativas e qualitativas, utilizaram-se, respectivamente, a análise de variância (Anova, com correção de Bonferroni, quando foi o caso) e o teste qui-quadrado. Foi feita a transformação logarítmica para variáveis quantitativas que não apresentaram distribuição normal. Os valores dos tercis foram utilizados para o agrupamento dos valores da variáveis renda familiar per capita.

As razões de prevalência (RP, brutas e ajustadas) de sobrepeso e obesidade foram obtidas a partir do modelo de Poisson, conforme recomendado por Barros e Hirakata (2003). Optou-se por construir modelos separados para as presenças de sobrepeso (versus normal) e obesidade (versus normal), considerando-se, como variáveis independentes, aquelas relativas à prática de atividade física (individualmente), independentemente da significância estatística. Em todos os casos, as RPs foram ajustadas a renda familiar per capita, escolaridade dos pais, sexo e idade da criança. A RP mede a prevalência da condição de interesse no grupo de expostos em relação à prevalência no grupo dos não expostos. Este método tem sido aplicado em estudos epidemiológicos transversais (BARROS; HIRAKATA, 2003).

Em todas as etapas da análise, utilizouse o programa para microcomputadores Stata, versão 10 (Stata statistical software release 10, College Station, TX Stata Corporation).

O projeto de pesquisa foi aprovado pelo Comitê de Ética em Pesquisa da Universidade Federal de Santa Catarina (parecer n. 031/06 de 24/04/06) e acompanha normas das Resoluções 196/96 e 251/97 do Conselho Nacional de Saúde sobre pesquisa envolvendo seres humanos.

\section{Resultados}

Nesta pesquisa, participaram 345 escolas, sendo 269 públicas e 76 particulares, e 4.964 escolares com idade de 6 a 10 anos, matriculados entre a $1^{\underline{a}}$ e a $4^{\underline{a}}$ série do ensino fundamental. Da amostra final, foram excluídos 275 escolares ausentes no dia da coleta dos dados antropométricos, 358 com idade acima de dez anos e 89 por inconsistência nos dados. Entre os 4.964 escolares que participaram da pesquisa, $2.375(47,8 \%)$ eram do sexo masculino. As prevalências de sobrepeso e obesidade foram de, respectivamente, 15,4\% (IC 95\%: 14,4\% - 16,5\%) e 6,1\% (IC 95\%: 5,4\% - 6,7\%), sendo estatisticamente semelhantes entre os sexos e categorias de idade. Os valores do IMC foram estatisticamente maiores para os meninos e entre as crianças mais velhas $(p<0,05)$ em comparação às demais.

A Tabela 1 apresenta os valores médios $(\mathrm{dp})$ e as frequências de crianças segundo estado nutricional e as variáveis de interesse. Verificaram-se associações estatisticamente significantes $(p<0,05)$ entre a presença de sobrepeso e obesidade para as variáveis frequência semanal de esportes que não aqueles feitos nas aulas de educação física (maior frequência entre crianças com obesidade em comparação àquelas com sobrepeso), horas despendidas por dia com computador durante a semana e nos finais de semana (ambos maiores entre crianças com sobrepeso do que para aquelas com obesidade), horas de sono/ noite (maior proporção de normais entre 


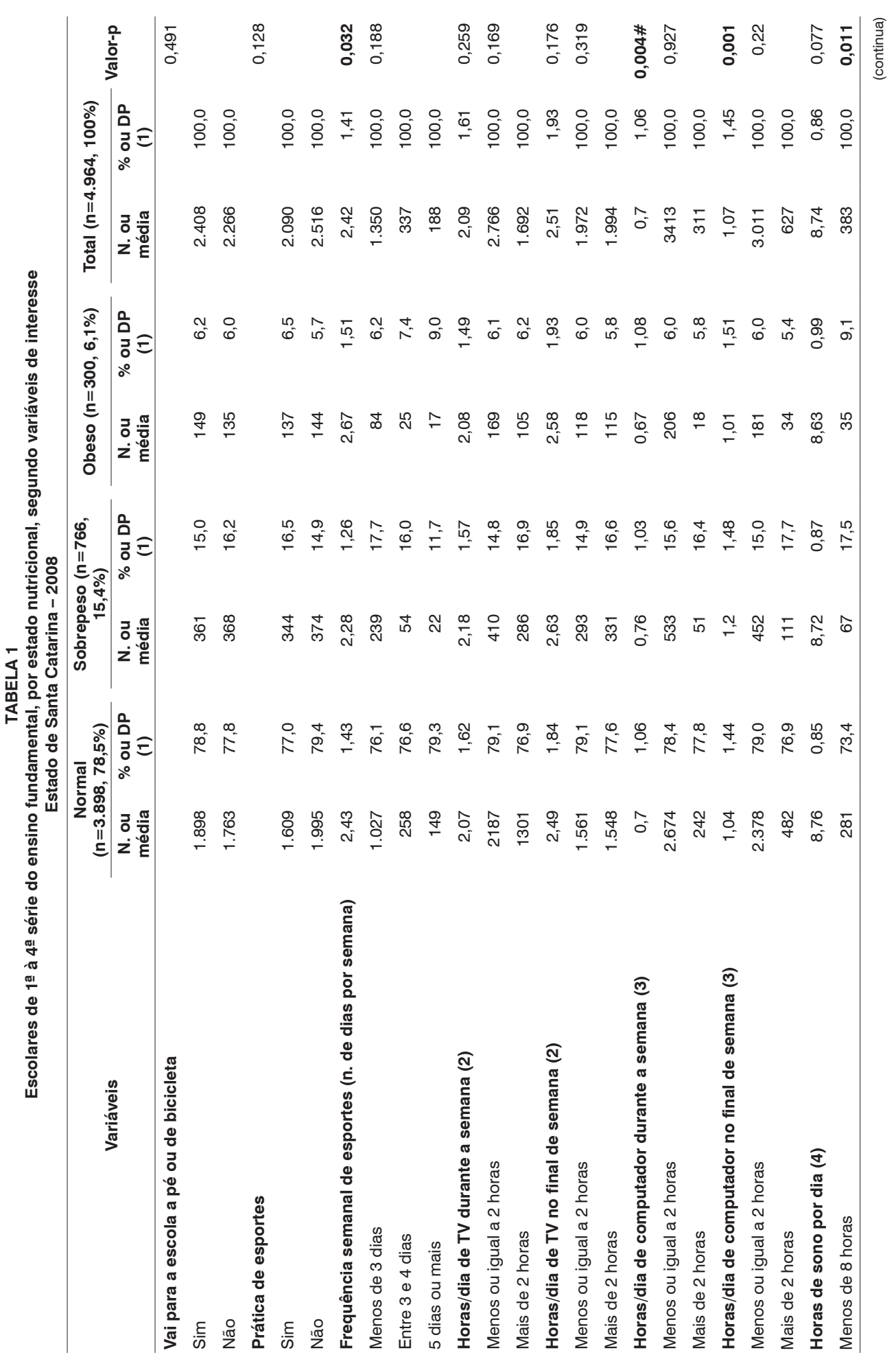




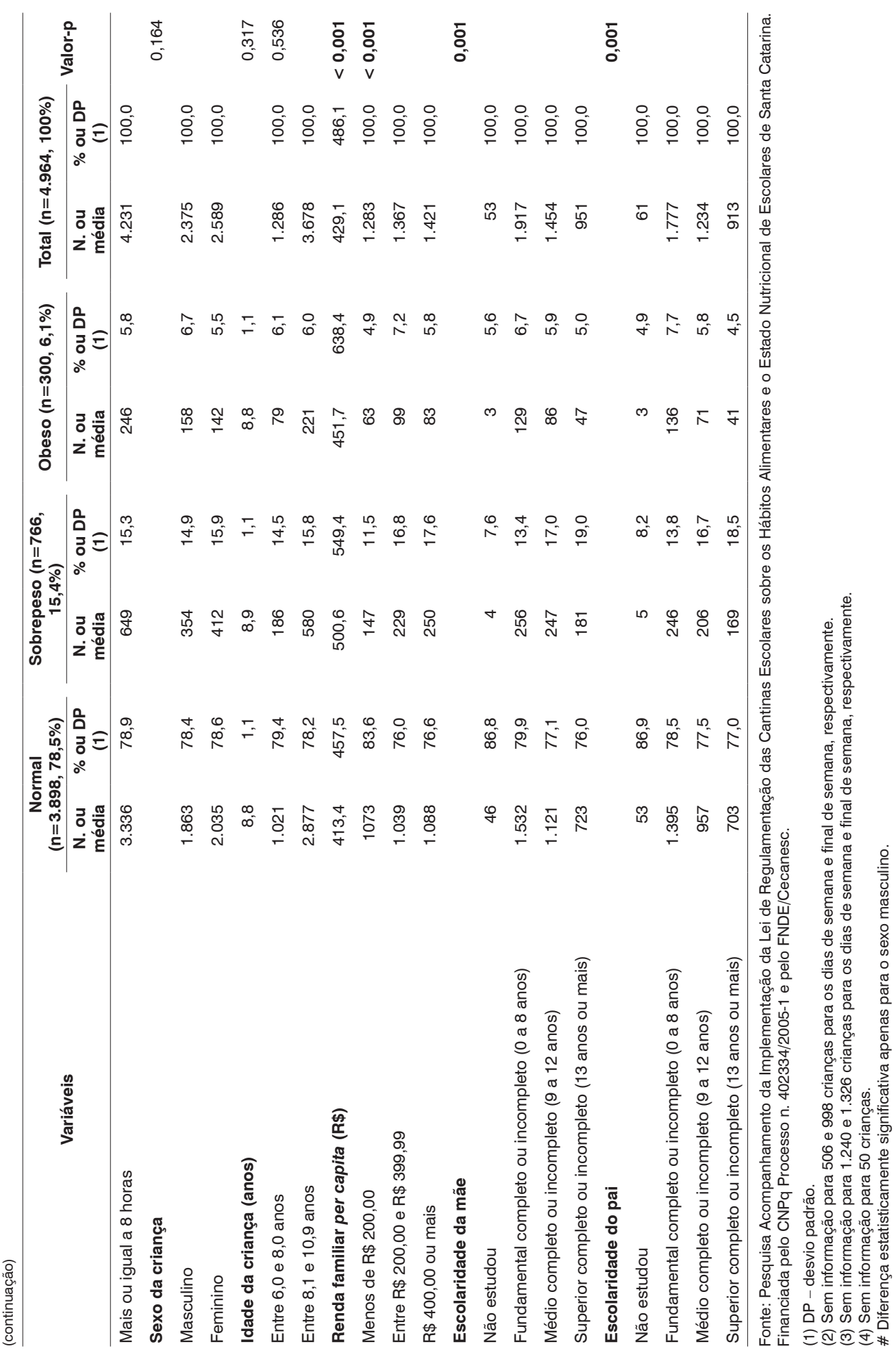


crianças com oito ou mais horas quando comparadas a crianças com menos de oito horas de sono/noite, enquanto a situação se inverte entre os escolares com soprepeso ou obesidade), renda per capita familiar e escolaridade dos pais.

Quanto ao tipo de atividade esportiva praticada pelas crianças, segundo estado nutricional e sexo, observou-se maior fre- quência de meninos com sobrepeso entre os que referiram praticar natação (Gráfico 1).

As razões de prevalência (brutas) para as presenças de sobrepeso e obesidade, segundo variáveis de controle sociodemográficas, encontram-se na Tabela 2. Associações estatisticamente significantes foram encontradas para algumas categorias da variável renda familiar per capita. Crianças

GRÁFICO 1

Porcentagem de escolares de $1^{\mathrm{a}}$ à $4^{\mathrm{a}}$ série do ensino fundamental, por sexo, segundo tipo de esporte praticado e estado nutricional Estado de Santa Catarina - 2008
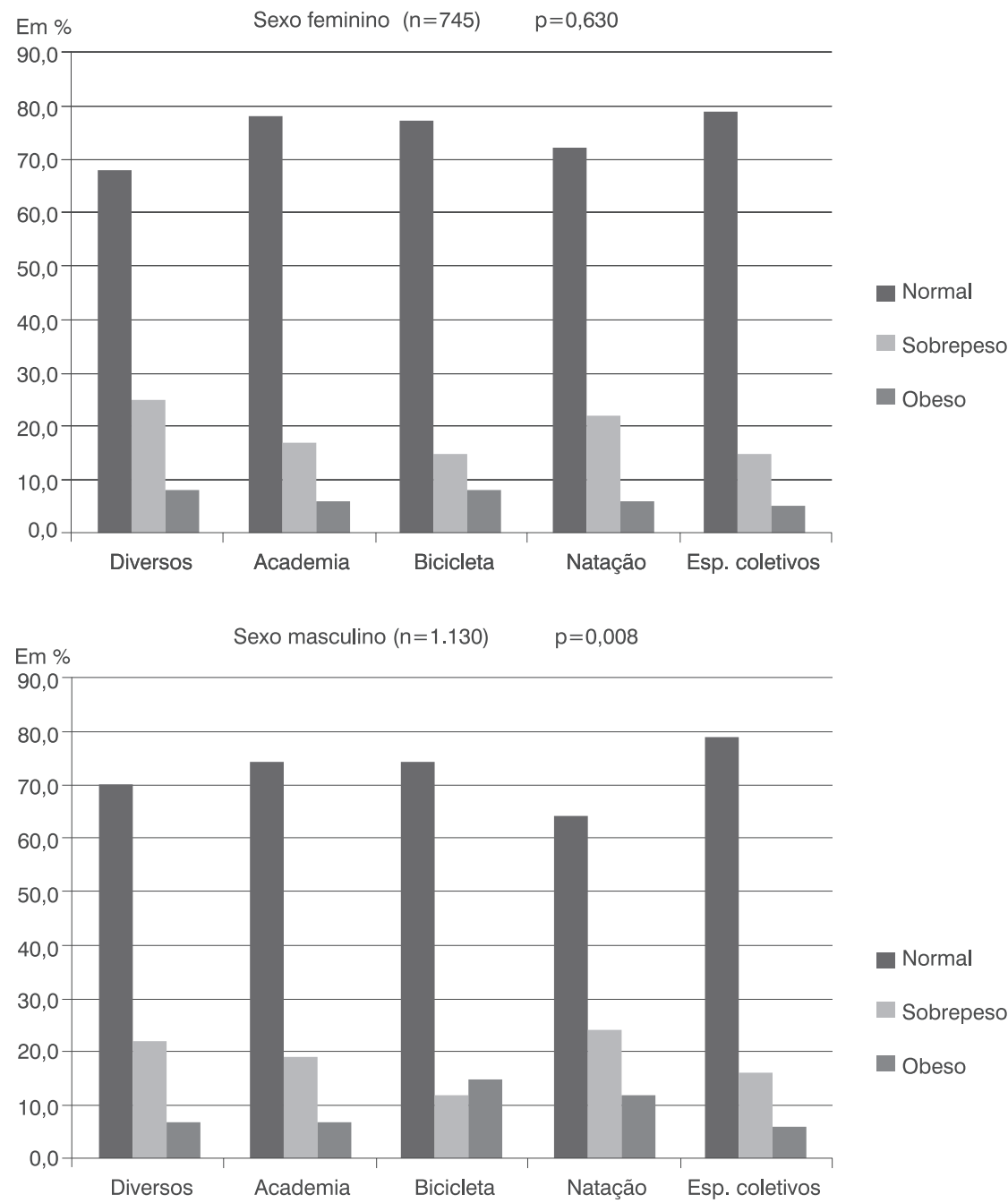

Fonte: Pesquisa Acompanhamento da Implementação da Lei de Regulamentação das Cantinas Escolares sobre os Hábitos Alimentares e o Estado Nutricional de Escolares de Santa Catarina. Financiada pelo CNPq Processo n. 402334/2005-1 e pelo FNDE/Cecanesc. 
TABELA 2

Razões de prevalência bruta para a presença de sobrepeso e obesidade em escolares de $1^{a}$ à $4^{\text {a }}$ série do ensino fundamental, segundo variáveis sociodemográficas Estado de Santa Catarina - 2008

\begin{tabular}{|c|c|c|c|c|}
\hline \multirow{2}{*}{ Variáveis } & \multicolumn{2}{|c|}{ Sobrepeso } & \multicolumn{2}{|c|}{ Obesidade } \\
\hline & $\mathbf{R P}$ & IC $95 \%$ & RP & IC $95 \%$ \\
\hline \multicolumn{5}{|l|}{ Sexo da criança } \\
\hline Masculino & 1 & & 1 & \\
\hline Feminino & 1,05 & $0,91-1,22$ & 0,83 & $0,67-1,05$ \\
\hline Idade da criança (anos) & 1,04 & $0,98-1,11$ & 0,98 & $0,91-1,05$ \\
\hline Entre 8,1 e 10,9 anos & 1 & & 1 & \\
\hline Menos ou igual a 8 anos & 1,09 & $0,92-1,28$ & 1,06 & $0,92-1,21$ \\
\hline Renda familiar per capita (R\$) & 1,01 & $1,01-1,01$ & 1 & $0,99-1,00$ \\
\hline Menos de $\mathrm{R} \$ 200,00$ & 1 & & 1 & \\
\hline Entre $R \$ 200,00$ e $R \$ 399,99$ & 1,5 & $1,22-1,84$ & 1,57 & $1,14-2,15$ \\
\hline $\mathrm{R} \$ 400,00$ ou mais & 1,55 & $1,26-1,90$ & 1,28 & $0,92-1,77$ \\
\hline \multicolumn{5}{|l|}{ Escolaridade da mãe } \\
\hline Não estudou & 0,9 & $0,74-1,09$ & 1,17 & $0,82-1,67$ \\
\hline Fundamental completo ou incompleto (0 a 8 anos) & 0,72 & $0,59-0,87$ & 1,27 & $0,91-1,78$ \\
\hline Médio completo ou incompleto (9 a 12 anos) & 0,4 & $0,14-1,08$ & 1 & $0,31-3,23$ \\
\hline Superior completo ou incompleto (13 anos ou mais) & 1 & & 1 & \\
\hline \multicolumn{5}{|l|}{ Escolaridade do pai } \\
\hline Não estudou & 0,91 & $0,75-1,12$ & 1,25 & $0,85-1,84$ \\
\hline Fundamental completo ou incompleto ( 0 a 8 anos) & 0,77 & $0,64-0,94$ & 1,61 & $1,14-2,29$ \\
\hline Médio completo ou incompleto (9 a 12 anos) & 0,44 & $0,18-1,08$ & 0,97 & $0,30-3,14$ \\
\hline Superior completo ou incompleto (13 anos ou mais) & 1 & & 1 & \\
\hline
\end{tabular}

Fonte: Pesquisa Acompanhamento da Implementação da Lei de Regulamentação das Cantinas Escolares sobre os Hábitos Alimentares e o Estado Nutricional de Escolares de Santa Catarina. Financiada pelo CNPq Processo n. 402334/2005-1 e pelo FNDE/Cecanesc.

oriundas de famílias com renda per capita entre $\mathrm{R} \$ 200,00$ e $\mathrm{R} \$ 400,00$ têm prevalência $50 \%$ maior de ter sobrepeso $(\mathrm{RP}=1,50, \mathrm{IC}$ 95\%: $1,22-1,84)$ e $57 \%$ maior de apresentar obesidade (RP $=1,57$, IC 95\%: 1,14-2,15), quando comparadas com aquelas de famílias com renda per capita abaixo de $\mathrm{R} \$$ 200,00 . A categoria de renda $R \$ 400,00$ ou mais mostrou-se associada apenas com o sobrepeso. Crianças de famílias com renda acima de $R \$ 400,00$ têm prevalência de sobrepeso $55 \%$ maior do que aquelas com renda inferior a $R \$ 200,0(R P=1,55, I C$ 95\%: 1,26-1,90).

$\mathrm{Na}$ Tabela 3 constam os valores das razões de prevalência para as presenças de sobrepeso e obesidade e as variáveis de controle atividade física, horas de TV/ computador e horas de sono/noite. Após ajuste, permaneceram associadas com o sobrepeso e a obesidade as variáveis frequência semanal de esportes que não aqueles feitos nas aulas de educação física $(\mathrm{RP}=1,12, \mathrm{IC} 95 \%: 1,02-1,23)$ e o número de horas de sono/noite $(\mathrm{RP}=1,56$, IC 95\%: 1,04-2,34). A variável horas despendidas por dia no computador nos finais de semana mostrou-se associada apenas com o sobrepeso (RP $=1,07, \mathrm{IC} 95 \%$ : 1.01-1,14).

\section{Discussão}

Os dados desta pesquisa indicaram prevalência de $15,4 \%$ de sobrepeso e $6,0 \%$ de obesidade. Valores semelhantes foram encontrados em levantamento realizado em escolas públicas e particulares na cidade de Florianópolis, capital do Estado de Santa Catarina, com escolares entre 7 e 10 anos de idade: $15,5 \%$ de sobrepeso e $5,5 \%$ de obesidade (ASSIS et al., 2005).

Em estudos internacionais que utilizaram o mesmo critério diagnóstico e faixa etária similar, as prevalências de sobrepeso e 
TABELA 3

Razões de prevalências (brutas e ajustadas) para a presença de sobrepeso e obesidade em escolares de $1^{\text {a }}$ à $4^{a}$ série do ensino fundamental, segundo variáveis relativas à atividade física Estado de Santa Catarina - 2008

\begin{tabular}{|c|c|c|c|c|c|c|c|c|}
\hline \multirow{3}{*}{ Variáveis } & \multicolumn{4}{|c|}{ Sobrepeso } & \multicolumn{4}{|c|}{ Obesidade } \\
\hline & \multicolumn{2}{|c|}{ Bruta } & \multicolumn{2}{|c|}{ Ajustada (1) } & \multicolumn{2}{|c|}{ Bruta } & \multicolumn{2}{|c|}{ Ajustada (1) } \\
\hline & $\mathbf{R P}$ & IC 95\% & $\mathbf{R P}$ & IC $95 \%$ & $\mathbf{R P}$ & IC $95 \%$ & $\mathbf{R P}$ & IC 95\% \\
\hline \multicolumn{9}{|l|}{$\begin{array}{l}\text { Vai para a escola a pé/ } \\
\text { bicicleta }\end{array}$} \\
\hline Sim & \multicolumn{2}{|l|}{1} & \multicolumn{2}{|l|}{1} & \multicolumn{2}{|l|}{1} & \multicolumn{2}{|l|}{1} \\
\hline Não & 1,08 & $0,93-1,25$ & 1,01 & $0,83-1,22$ & 0,98 & $0,83-1,15$ & 0,9 & $0,73-1,11$ \\
\hline \multicolumn{9}{|l|}{ Prática de esportes } \\
\hline Não & \multicolumn{2}{|l|}{1} & \multicolumn{2}{|l|}{1} & \multicolumn{2}{|l|}{1} & \multicolumn{2}{|l|}{1} \\
\hline Sim & 0,9 & $0,77-1,04$ & 1,02 & $0,85-1,22$ & 0,86 & $0,73-1,01$ & 0,82 & $0,67-1,01$ \\
\hline $\begin{array}{l}\text { Frequência semanal de } \\
\text { esporte (n. de dias por } \\
\text { semana) }\end{array}$ & 0,94 & $0,86-1,02$ & 0,94 & $0,85-1,05$ & 1,1 & $1,02-1,19$ & 1,12 & $1,02-1,23$ \\
\hline Menos de 3 dias & 1,35 & $0,82-2,21$ & 1,5 & $0,79-2,83$ & 0,86 & $0,56-1,33$ & 0,94 & $0,57-1,54$ \\
\hline Entre 3 e 4 dias & 1,47 & $0,95-2,27$ & 1,54 & $0,87-2,74$ & 0,74 & $0,51-1,07$ & 0,73 & $0,47-1,12$ \\
\hline 5 dias ou mais & \multicolumn{2}{|l|}{1} & \multicolumn{2}{|l|}{1} & \multicolumn{2}{|l|}{1} & \multicolumn{2}{|l|}{1} \\
\hline $\begin{array}{l}\text { Horas/dia de TV durante a } \\
\text { semana }\end{array}$ & 1,03 & $0,99-1,09$ & 1,02 & $0,97-1,08$ & 1,01 & $0,95-1,05$ & 1 & $0,94-1,06$ \\
\hline Mais de 2 horas & \multicolumn{2}{|l|}{1} & \multicolumn{2}{|l|}{1} & \multicolumn{2}{|l|}{1} & \multicolumn{2}{|l|}{1} \\
\hline Menos ou igual a 2 horas & 1,05 & $0,79-1,40$ & 1,01 & $0,84-1,21$ & 0,97 & $0,69-1,36$ & 0,77 & $0,50-1,16$ \\
\hline $\begin{array}{l}\text { Horas/dia de TV no final de } \\
\text { semana }\end{array}$ & 1,03 & $0,99-1,08$ & 1,01 & $0,96-1,07$ & 1,01 & $0,95-1,05$ & 1,01 & $0,95-1,07$ \\
\hline Mais de 2 horas & \multicolumn{2}{|l|}{1} & \multicolumn{2}{|l|}{1} & \multicolumn{2}{|l|}{1} & \multicolumn{2}{|l|}{1} \\
\hline Menos ou igual a 2 horas & 1,14 & $0,98-1,33$ & 1,03 & $0,85-1,24$ & 1,04 & $0,88-1,24$ & 0,96 & $0,78-1,19$ \\
\hline $\begin{array}{l}\text { Horas/dia de computador } \\
\text { (semana) }\end{array}$ & 1,05 & $0,97-1,12$ & 1,02 & $0,93-1,12$ & 0,97 & $0,89-1,07$ & 0,89 & $0,79-1,00$ \\
\hline Mais de 2 horas & \multicolumn{2}{|l|}{1} & \multicolumn{2}{|l|}{1} & 1 & & 1 & \\
\hline Menos ou igual a 2 horas & 1,05 & $0,79-1,40$ & 1,07 & $0,77-1,51$ & 0,97 & $0,69-1,39$ & 0,77 & $0,50-1,16$ \\
\hline $\begin{array}{l}\text { Horas/dia computador (final } \\
\text { semana) }\end{array}$ & 1,06 & $1,01-1,12$ & 1,07 & $1,01-1,14$ & 0,99 & $0,92-1,05$ & 0,91 & $0,83-0,99$ \\
\hline Mais de 2 horas & 1 & & 1 & & 1 & & 1 & \\
\hline Menos ou igual a 2 horas & 1,17 & $0,95-1,44$ & 1,22 & $0,95-1,56$ & 0,93 & $0,72-1,21$ & 0,72 & $0,52-0,99$ \\
\hline Horas sono/noite & 0,96 & $0,88-1,04$ & 0,91 & $0,83-1,01$ & 0,87 & $0,78-0,98$ & 0,88 & $0,80-0,97$ \\
\hline Mais de 8 horas & 1 & & 1 & & 1 & & 1 & \\
\hline Menos ou igual a 8 horas & 1,18 & $0,94-1,48$ & 1,36 & $1,05-1,75$ & 1,61 & $1,15-2,25$ & 1,56 & $1,04-2,34$ \\
\hline
\end{tabular}

Fonte: Pesquisa Acompanhamento da Implementação da Lei de Regulamentação das Cantinas Escolares sobre os Hábitos Alimentares e o Estado Nutricional de Escolares de Santa Catarina. Financiada pelo CNPq Processo n. 402334/2005-1 e pelo FNDE/Cecanesc.

(1) Renda familiar per capita, escolaridade dos pais, sexo e idade da criança.

obesidade encontradas variaram entre 3,8\% e $28 \%$, em países como França, Alemanha, Itália, Portugal e México (WHO, 2007; APFELBACHER et al., 2008; MORAES et al., 2004; FERREIRA; MARQUES-VIDAL, 2008).

No Brasil, nas últimas décadas, inquéritos nacionais relataram que a presença de sobrepeso e de obesidade em crianças e adolescentes aumentou de $4,9 \%$ para $17,4 \%$, entre 1974 e 1997. Dados provenientes da pesquisa Nacional sobre Saúde e Nutrição indicam que cerca de 1,5 milhão de crianças são portadoras de obesidade, com maior frequência em meninas, sendo que nas Regiões Sul e Sudeste sua ocorrência é o dobro daquela observada no Nordeste, com prevalência de obesidade mais elevada nas faixas de renda intermediárias e alta (WANG et al., 2002;TRICHES; GIULIANI, 2005).

Nesta pesquisa. o sobrepeso e a obesidade foram associados com a frequência 
semanal de esportes que não aqueles feitos nas aulas de educação física e com o número de horas de sono/noite. Além disso, o sobrepeso foi associado com as horas gastas por dia no computador nos finais de semana.

A atividade física tem sido identificada como fator protetor contra o sobrepeso e a obesidade, pois crianças mais ativas tendem a apresentar menor percentual de gordura corporal, enquanto crianças com obesidade, quando comparadas com crianças eutróficas, são menos ativas e participam menos de atividades intensas e moderadas, com predomínio das atividades de baixa intensidade (LAZZER et al., 2003). Nas últimas décadas, vem diminuindo o nível de atividade física devido ao aumento no uso de veículos motores para deslocamento até a escola, à menor participação em esportes organizados e ao maior tempo gasto em atividades sedentárias, como aquelas que envolvem TV, computador e video game (CDC, 2005).

Estudos nacionais e internacionais mostram que, nas últimas décadas, há uma tendência de declínio na prática de atividade física e prevalência elevada de inatividade física na população jovem (FARIAS JUNIOR, 2000; GUEDES et al., 2001; CANTERA-GARDE; DEVÍS-DEVÍS, 2000). Em escala mundial, estima-se uma proporção de inatividade física entre 31\% e $51 \%$ da população, com uma média de $41 \%$, sendo que na região das Américas mais de $50 \%$ dos habitantes são sedentários (OMS, 2002).

Estudos revelam uma relação inversa entre o nível de atividade física e a obesidade, sendo verificado alto grau de sedentarismo, decorrente do elevado número de horas diante da televisão e com jogos eletrônicos e do reduzido número de horas dedicadas ao esporte e jogos ativos (PIMENTA; PLAMA, 2001; PAJUELO et al., 2005). Para a garantia de uma vida saudável, a recomendação é realizar 60 minutos diários de atividade física moderada e intensa, de maneira acumulativa, em horas de educação física, recreação, esportes fora da escola, antes e depois dos programas da escola (BARUK et al., 2006).
Para a American Academy of Pediatrics (2001), o tempo gasto por crianças e adolescentes diante da TV e com jogos eletrônicos não deve ultrapassar duas horas por dia. Estudos de base populacional realizados no Brasil indicam condições muito aquém das recomendadas. Os resultados desta pesquisa mostram um percentual elevado de escolares que permanecem mais de duas horas em frente à televisão e ao computador durante o dia. O Framinggham Children's Study, estudo longitudinal de crianças com idades entre 4 e 11 anos, confirma maiores valores de IMC no grupo que assistiu mais à televisão ( $\geq 3$ horas/dia) e menores valores no grupo que assistiu menos ( $<1,75$ hora/dia). A correlação moderada e inversa verificada entre brincar e/ou praticar exercícios com assistir televisão ou ocupar-se com jogos eletrônicos, em ambos os sexos, evidencia que crianças mais sedentárias assistem mais à televisão do que aquelas mais ativas, estando mais expostas ao ganho de peso corporal (CAMPANHOLO et al., 2008).

Em países desenvolvidos e em desenvolvimento, estudos longitudinais revelaram uma relação de causalidade entre horas de TV e obesidade. Gortmaker e colaboradores (1996), por meio de um estudo de coorte, confirmaram que assistir mais de cinco horas de TV por dia, em relação a assistir duas horas ou menos, foi fator etiológico para o desenvolvimento da obesidade e não por inabilidade física ou isolamento social. O hábito de assistir TV precocemente pode levar a criança a se tornar obesa, agravando, assim, o sedentarismo pela capacidade limitada de fazer exercícios físicos e também pelo isolamento social, que aumenta a chance daquele hábito ser a alternativa mais predominante de lazer (CAMPANHOLO et al., 2008).

Estudos pontuais desenvolvidos em escolas de ensino público e privado no Brasil revelaram que a obesidade está associada significativamente com escolaridade elevada dos pais, alta renda familiar e presença nas residências de TV, computador, telefone e video game, além de mostrarem que crianças eutróficas são mais ativas, praticam atividades físicas mais intensas e gastam 
menos tempo assistindo à televisão e jogando video games do que aquelas com excesso de peso (OLIVEIRA et al., 2003; BARUK et al., 2006).

Portanto, assistir televisão por mais de três horas/dia e jogar video game por mais de duas horas/dia são fatores de risco para sobrepeso e obesidade.

Além destes fatores, estudos epidemiológicos têm procurado correlacionar a curta duração do tempo de sono com o aumento do Índice de Massa Corporal (IMC), evidenciando que a diminuição no tempo dedicado ao sono é fator predisponente para o aparecimento da obesidade (CRISPIM et al., 2007).

Em 2000, foi realizada uma pesquisa como parte do projeto Previne (Programa de Estudos e Vigilância Nutricional de Escolares), desenvolvido pela Universidade Católica de Brasília (UCB), envolvendo 452 escolares de ambos os sexos, na faixa etária de 6 a 10 anos. A ocorrência de obesidade foi semelhante nos dois sexos, atingindo $21,1 \%$ dos meninos e $22,9 \%$ das meninas. A tendência ao sedentarismo foi associada ao tempo de permanência sentado e à porcentagem de gordura corporal nas crianças. Foi observado também que o número de horas de sono diárias pode favorecer a redução da gordura corporal em crianças, evidenciando que o sono pode atuar favoravelmente na manutenção da composição corporal em crianças e deveria ser estimulado, principalmente nos casos de sobrepeso e obesidade (GIULIANI; CARNEIRO, 2004).

Uma pesquisa na China revelou que as crianças que relataram entre 9 e 11 horas de sono por noite apresentaram peso normal, após ajuste para idade, sexo e outros fatores de risco, assim como crianças cujos responsáveis dormiam menos e filhos de mães com nível educacional mais elevado ou que dormiam com os pais tinham menos tempo de sono noturno do que outras crianças (FAN et al., 2009).

Outros levantamentos nacionais e internacionais também constataram que crianças e adolescentes obesos dormiam menos do que aquelas não portadoras de obesidade, sendo que, para cada hora de sono perdido, a chance de ter obesidade aumentava $80 \%$, existindo uma relação dose-resposta entre horas de sono e obesidade para crianças que dormiam menos de oito horas, em comparação com aquelas que dormiam mais de dez horas, bem como uma correlação inversa e significativa entre o percentual de gordura corporal e o tempo diário médio de sono (GUPTA et al., 2002; SEKINE et al., 2002; GUIGLIANI; CARNEIRO, 2004; GUIMARÃES et al., 2006).

As prevalências de sobrepeso e de obesidade encontradas entre os escolares do Estado de Santa Catarina foram associadas a fatores como sedentarismo, ocasionado pela facilidade de acesso aos jogos eletrônicos e à televisão, aliados à diminuição da prática de atividade física e da duração das horas de sono, elementos que podem interferir na qualidade de vida dos mesmos.

Estudos com adolescentes mostraram associação significativa entre baixa qualidade de vida relacionada à saúde e obesidade (PINHAS-HAMIEL, 2006; SCHWIMMER, BURWINK; VARNI, 2003; ZELLER; MODI, 2006). Considerando o fato de que, atualmente, atingir uma idade avançada é uma realidade populacional também nos países mais pobres, há necessidade de incentivar programas de prevenção nos cuidados de atenção integral à saúde, principalmente de crianças na idade escolar, pois a prevenção é efetiva em qualquer fase da vida.

As doenças crônicas e suas consequências podem ser evitadas, proporcionado redução nas taxas de morbidade e mortalidade e aumento na longevidade da população. É preciso evitar a existência de uma população que apresenta longevidade elevada, porém comprometida por doenças associadas ao excesso de peso iniciado na idade escolar e que persistiu ao longo da vida (NORONHA; FIGUEIREDO; ANDRADE, 2010; VERAS, 2009).

A escola é um ambiente propício para a promoção de informação e esclarecimentos aos pais dos escolares a respeito da relevância da prática de atividade física e controle do número de horas da criança em frente a aparelhos eletrônicos e televisão, bem como 
da importância da quantidade adequada de horas de sono, uma vez que estes fatores estão relacionados com o desenvolvimento de sobrepeso e obesidade.

Entende-se que de uma forma mais ampla, no contexto da sociedade e das práticas comunitárias, o papel do Estado é também essencial na medida em que deveria garantir a existência de espaços públicos adequados e seguros à prática da atividade física, espaços estes cada

\section{Referências}

AMERICAN ACADEMY OF PEDIATRICS. Children, adolescents and television. Pediatrics, Elk Grove Village, v. 107, p. 423-6, 2001.

APFELBACHER, C. J.; LOERBROKS, A.; CAIRNS, J.; BEHRENDT, H.; RING, J.; KRÄMER, U. Predictors of overweight and obesity in five to seven-year-old children in Germany: results from cross-sectional studies. BMC Public Health, Londres, v. 21, p. 171-178, 2008.

ASSIS, M. A.; ROLLAND-CACHERA, M. F.; GROSSEMAN, S.; VASCONCELOS, F. A. G.; LUNA, M. E.; CALVO, M. C. Obesity, overweight and thinness in schoolchildren of the city of Florianópolis, Southern Brazil. European Journal Clinical Nutrition, Londres, v. 59, n. 9, p. 1015-1021, 2005.

BARROS, A. J. D.; HIRAKATA, V. N. Alternatives for logistic regression in crosssectional studies: an empirical comparison of models that directly estimate the prevalence ratio. BMC Medical Research Methodology, Londres, v. 3, p. 21-34, 2003.

BARUK, S. B. S.; ROSADO, L. E. F. P. L.; ROSADO, G. P.; RIBEIRO, R. C. Associação entre estado nutricional e atividade física em escolares da rede municipal de ensino de Corumbá-MS. Revista Brasileira de Medicina do Esporte, São Paulo, v. 12, n. 2, p. 90-94, 2006.

BATISTA FILHO, M.; SOUZA, A. I.; MIGLIOLI, T. C.; SANTOS, M. C. Anemia e obesidade: um paradoxo da transição nutricional brasi- vez mais raros hoje em dia, diante do crescimento desordenado das cidades e da insegurança causada pela violência, que impede os jovens e crianças de passarem mais tempo ao ar livre em práticas comuns no passado, como jogar bola e andar de bicicleta, segregando-os cada vez mais a espaços fechados, domésticos, onde normalmente a única atividade disponível se reduz aos aparelhos de TV, video games e computadores.

leira. Cadernos de Saúde Pública, Rio de Janeiro, v. 24 (suppl. 2), p. 247-257, 2008.

BAUGHCUM, A. E.; CHAMBERLIN, L. A.; DEEKS, C. M.; POWERS, S. W.; WHITAKER, R. C. Maternal perceptions of overweight preschool children. Pediatrics, v.106, n. 6, p.1380-1386, 2000.

CAMPAGNOLO, P. D. B.; VITOLO, M. R.; GAMA, C. M. Fatores associados ao hábito de assistir TV em excesso entre adolescente.

Revista Brasileira de Medicina do Esporte, São Paulo, v. 14, n. 3, p. 197-200, 2008.

CANTERA-GARDE, M. A.; DEVÍS-DEVÍS, J. Physical activity levels of secondary school Spanish adolescent. European Journal Physical Education, v. 5, p. 28-44, 2000.

CENTER FOR DISEASE CONTROL AND PREVENTION. Youth risk behavior surveillance-United State, 2005. Surveillance summaries. MMWR, v. 55, n. SS-5, 2006.

Barriers to children walking or from School-United State, 2004. MMWR - Morbidity and Mortality Wkeely Report, Biolnfo Bank Institut, v. 54, n. 38, p. 949952, 2005.

COLE, T. J.; BELLIZZI, M. C.; FLEGAL, K. M.; DIETZ, W. H. Establishing a standard definition for child overweight and obesity worldwide:international survey. British Medical Journal, Londres, v. 6, n. 320, p. 1240-1243, 2000.

CRISPIM, C. A.; ZALCMANN, I.; DÁTTILA, M.; PADRILHA, H. G.; TUFIK, S.; MELLO, 
M. T. Relação entre sono e obesidade: uma revisão de literatura. Arquivos Brasileiros de Endocrinologia e Metabolismo, São Paulo, v. 51, n. 7, p.1041-1049, 2007.

DANADIAN, K.; LWY, V.; JANOSKY, J. J.; ARSLANIAN, S. Lipolysis in AfricanAmerican children: is it a metabolic risk factor predisposing to obesity? Journal Clinical Endocrinology Metabolism, Chevy Chase, MD, v. 87, n. 7, p. 3022-3026, 2001.

FAN, J.; SHANKUAN, Z.; CHONGHUAI, Y.; XINGMING, J. I. N.; HARI, B.; XIAOMING, S. Sleep and obesity in preschool children. Journal of Pediatrics, Philadelphia, v. 150, p. 814-818, 2009.

FARIAS JUNIOR, J. C. Prevalência e fatores de influência para inatividade física em adolescentes. Revista Brasileira de Ciência e Movimento, Taguatinga, CE, v. 14, p.57-64, 2006.

FERREIRA, R. J.; MARQUES-VIDAL, P. M. Prevalence and determinants of obesity in children in public schools of Sintra, Portugal. Obesity Research, v. 16, n. 2, p. 497-500, 2008.

GABRIEL, C. G.; VASCONCELOS, F. A. G.; ANDRADE, D. F.; SCHMITZ, B. A. S. First Law regulating school canteens in Brazil: evaluation after seven years of implementation. Archivos Latinoamericanos de Nutrición, Caracas, v. 59, n. 2, p. 128-138, 2009.

GIUGLIANI, R.; CARNEIRO, E. C. Fatores associados à obesidade em escolares. Jornal de Pediatria, São Paulo, v. 80, n. 1, p. 17-22, 2004.

GOTMAKER, S. L.; MUST, A.; SOBOL, A. M.; PETERSON, K.; COLDITZ, G. A.; DIETZ, W. H. Television viewing as a cause of increasing obesity among children in the United States, 1986-1990. Archivos Pediatrics Adolescent Medicine, Genebra, v. 150, p. 356-362, 1996.

GRUPTA, N. K.; MUELLER, W. H.; CHAN, W.; MEININGER, J. C. Is obesity associated with poor sleep quality in adolescents? American Journal Human Biology, Los Angeles, v. 8, p. 714-762, 2002.
GUEDES, D. P.; GUEDES, J. E. R. P.; BAROSA, D. F.; OLIVEIRA, J. A. Níveis de prática de atividade física habitual em adolescentes. Revista Brasileira de Medicina do Esporte, São Paulo, v. 7, p. 187-200, 2001.

GUIMARÃES, L. V.; BARROS, M. B. A.; MARTINS, M. S. A. S.; DUARTE, E. C. Fatores associados ao sobrepeso em escolares. Revista de Nutrição, Campinas, v. 19 , n. 1 , p. 5-17, 2006

IBGE - Instituto Brasileiro de Geografia e Estatística. Estados. Santa Catarina. E/DPE/ COPIS. 2008.

LAZZER, S.; BOIRIE, Y.; BITAR, A.; MONTAURER, C.; VERNET, J.; MEYER, M. Assessment of energy expenditure associated with physical activities in freeliving obese an non-obese adolescents. American Journal Clinical Nutrition, Bethseda, MD, v. 78, p. 471-479, 2003.

LIMA, S. C.; ARRAIS, R. F.; PEDROSA, L. F. C. Avaliação da dieta habitual de crianças e adolescentes com sobrepeso e obesidade. Revista de Nutrição, Campinas, v. 17, p. 469-477, 2004

LOHMAN, T. G.; ROCHE, A. F.; MARTORELL, R. Anthropometric Standardization Reference Manual. Human Kinetics Books, Champaign, Illinois, 1991.

MORAES, S. A.; BELTRÁN ROSAS, J.; MONDINI, L.; FREITAS, I. C. Prevalência de sobrepeso e obesidade e fatores associados em escolares de área urbana de Chilpancingo, Guerrero, México, 2004. Cadernos de Saúde Publica, Rio de Janeiro, v. 22, n. 6, p.1289-1301, 2006.

NORONHA, K.; FIGUEIREDO, L.; ANDRADE, $M$. V. Health and economic growth among the states of Brazil from 1991 to 2000. Revista Brasileira de Estudos de População, v. 27, n. 2, p. 269-283, 2010.

OLIVEIRA, A. M. A.; CERQUEIRA, E. M. M.; SOUZA, J. S.; OLIVEIRA, A. C. Sobrepeso e obesidade infantil: influência de fatores biológicos e ambientais em Feira de Santana, BA. Arquivos Brasileiros de Endocrinologia e Metabolismo, São Paulo, v. 47, n. 2, p. 144-150, 2003. 
PAJUELO, J.; BERNUI, I.; QUIROZ, G.; QUISPE, J. Características alimentares y horas de ver televisón en niñas de 6 años, obesas e normales. Consensus, v. 9, n. 10, p. 9-16, 2005.

PIMENTA, A. P. A.; PLAMA, A. Perfil epidemiológico da obesidade em crianças: relação entre televisão, atividade física e obesidade. Revista Brasileira de Ciência e Movimento, Taguatinga, CE, v. 9, p.1924, 2001.

PINHAS-HAMIEL, O.; SINGER, S.; PILPEL, N.; FRADKIN, A.; MODAN, D.; REICHMAN, B. Health-related quality of life among children and adolescents: associations with obesity. Int. J. Obes. (Lond.), v. 30, n. 2, p. 267-72, 2006.

RIBEIRO, I. C.; TADDEI, J. A. A. C.; COLUGNATTI, F. Obesity among childeren attending elementary public schools in São Paulo, Brazil: a case-control study. Public Health Nutrition, v. 6, n. 7, p. 659-663, 2003.

RICARDO, G. D.; CALDEIRA, G. V.; CORSO, A. C. T. Prevalência de sobrepeso e obesidade e indicadores de adiposidade central em escolares de Santa Catarina, Brasil. Revista Brasileira de Epidemiologia, São Paulo, v. 12, n. 3, p. 424-435, 2009.

SCHWIMMER, J. B.; BURWINKLE, T. M.; VARNI, J. W. Health-related quality of life of severely obese children and adolescents. JAMA, v. 289, n, 14, p. 813-9, 2003.

SEKINE, M.; YAMAGAMI, T.; HANDA, K.; SAITO, T.; NANRI, S.; KAWAMINAMI, K. A dose-response relationship between sort sleeping hours and childhood obesity: results of the Toyama Birth Cohort Study. Child Care Helath Development, Oxford, v. 28, p.70-163, 2002.
TRICHES, R. M.; GIUGLIANI, E. R. Obesidade, práticas alimentares e conhecimentos de nutrição em escolares. Revista de Saúde Pública, Rio de Janeiro, v. 39, p. 541-547, 2005.

VERAS, R. Envelhecimento populacional contemporâneo: demandas, desafios e inovações. Rev. Saúde Pública, v. 43, n. 3, p.548-554, 2009.

WANG, Y.; MONTEIRO, C. A.; POPKIN, B. $M$. Trends of obesity and underweight in older children and adolescents in the United States, Brazil, China, and Russia. American Journal Clinical Nutrition, Bethseda MD, v. 75, n. 6, p. 971-977, 2002.

WORLD HEALTH ORGANIZATION EUROPEAN. The challenge of obesity in the WHO European Region and the strategies for response. Editado por Francesco Branca, Haik Nikogosian and Tim Lobstein, 2007.

WORLD HEALTH ORGANIZATION. Global strategy on diet, physical activity and health. Geneva: WHO, 2004. (Fifty-Seventh World Health - WHA 57.17).

. Obesity: preventing and managing

the global epidemic. Tecnhical Report Séries 894. Geneve, 1998.

The world health report 2002.

Reducing risks, promoting healthy life. Geneva, 2002.

ZELLER, M. H.; MODI, A. C. Predictors of health-related quality of life in obese youth. Obesity (Silver Spring), v. 14, n. 1 p. 122 30, 2006.

\section{Resumen}

Factores de comportamiento asociados al sobrepeso y a la obesidad en escolares del Estado de Santa Catarina

Se realizó un estudio transversal con 4.964 escolares de enseñanza fundamental del Estado de Santa Catarina para verificar la asociación entre las variables socioeconómicas y de actividad física, con el sobrepeso y la obesidad, estos últimos diagnosticados a partir del Índice de Masa 
Corporal (IMC). Se estimaron como variables de resultados, por punto y por intervalo con un $95 \%$ de confianza, las prevalencias de sobrepeso y obesidad. Las razones de prevalencia se obtuvieron por el modelo de Poisson y se ajustaron a la renta familiar per capita, escolaridad de los padres, sexo y edad del niño. Las prevalencias de sobrepeso y obesidad fueron $15,4 \%$ y $6,1 \%$, respectivamente, semejantes entre sexo y categorías de edad. Tras el ajuste, las variables asociadas con el sobrepeso y obesidad fueron: frecuencia semanal de deportes; número de horas de sueño/noche; y horas/día en el ordenador (fin de semana). Reducida práctica de deportes, menor duración de sueño/noche y más tiempo frente al ordenador se asocian con el sobrepeso y obesidad en estos escolares.

Palabras-clave: Niño. Estado nutricional. Escuela. Exceso de peso.

\section{Abstract \\ Behavioral factors associated with overweight and with obesity in students in the State of Santa Catarina}

A cross-sectional study with 4,964 elementary school students of the State of Santa Catarina was performed to check for the association of socioeconomic variables and exercise with overweight or obesity, the two latter diagnosed by the Body Mass Index (BMI). The outcome variables, overweight and obesity, were estimated by point prevalence and $95 \%$ confidence intervals. Prevalence ratios were obtained by the Poisson model and adjusted to per capita family income, parents' schooling, child's gender and age. The prevalence of overweight and obesity were $15.4 \%$ and $6.1 \%$, respectively, similar between gender and age categories. After adjustments, variables associated with overweight and obesity were: weekly frequency of practicing sports; number of hours of sleep/night; and hours/day on the computer (weekends). Less sports practice, less hours of sleep/night and more time on the computer were associated with overweight or obesity in the students studied.

Keywords: Children. Nutritional status. School. Weight excess.

Recebido para publicação em 04/04/2011

Aceito para publicação em 07/01/2012 